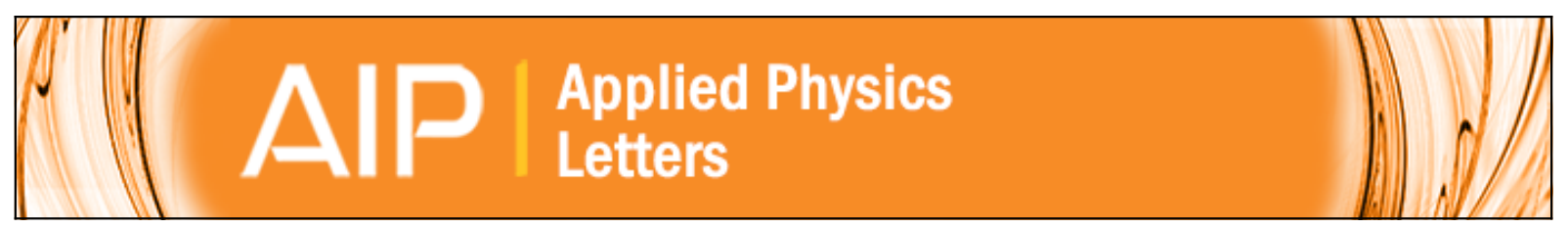

\title{
Surface and interface states of Bi2Se3 thin films investigated by optical second- harmonic generation and terahertz emission
}

S. Y. Hamh, S.-H. Park, S.-K. Jerng, J. H. Jeon, S. H. Chun, J. H. Jeon, S. J. Kahng, K. Yu, E. J. Choi, S. Kim, S.-H. Choi, N. Bansal, S. Oh, Joonbum Park, Byung-Woo Kho, Jun Sung Kim, and J. S. Lee

Citation: Applied Physics Letters 108, 051609 (2016); doi: 10.1063/1.4941420

View online: http://dx.doi.org/10.1063/1.4941420

View Table of Contents: http://scitation.aip.org/content/aip/journal/apl/108/5?ver=pdfcov

Published by the AIP Publishing

\section{Articles you may be interested in}

Aging and reduced bulk conductance in thin films of the topological insulator $\mathrm{Bi} 2 \mathrm{Se} 3$

J. Appl. Phys. 113, 153702 (2013); 10.1063/1.4801911

Modulation of external electric field on surface states of topological insulator Bi2Se3 thin films

Appl. Phys. Lett. 101, 223109 (2012); 10.1063/1.4767998

Significant second-harmonic generation in two lead-free polar oxides BilnO 3 and BiAlO 3 : A first-principles investigation

Appl. Phys. Lett. 94, 191908 (2009); 10.1063/1.3136838

Spectroscopic second harmonic generation measured on plasma-deposited hydrogenated amorphous silicon thin films

Appl. Phys. Lett. 85, 4049 (2004); 10.1063/1.1812836

Second-harmonic generation study of domain walls in x Bi 2 Ti 4 O 11 -(1-x) Bi 4 Ti 3012 films with large dielectric permittivity

J. Appl. Phys. 91, 3172 (2002); 10.1063/1.1445497

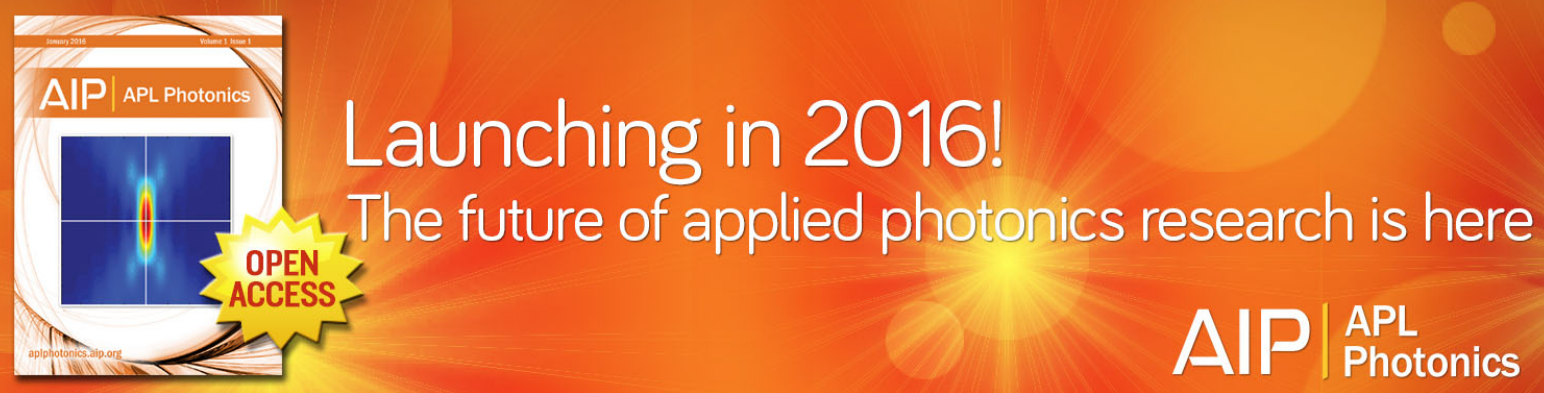




\title{
Surface and interface states of $\mathrm{Bi}_{2} \mathrm{Se}_{3}$ thin films investigated by optical second-harmonic generation and terahertz emission
}

\author{
S. Y. Hamh, ${ }^{1}$ S.-H. Park, ${ }^{1}$ S.-K. Jerng, ${ }^{2}$ J. H. Jeon, ${ }^{2}$ S. H. Chun, ${ }^{2}$ J. H. Jeon, ${ }^{3}$ S. J. Kahng, ${ }^{3}$ \\ K. Yu, ${ }^{4}$ E. J. Choi, ${ }^{4}$ S. Kim, ${ }^{5}$ S.-H. Choi, ${ }^{5}$ N. Bansal, ${ }^{6}$ S. Oh, ${ }^{7}$ Joonbum Park, ${ }^{8}$ \\ Byung-Woo Kho, ${ }^{8}$ Jun Sung Kim, ${ }^{8}$ and J. S. Lee ${ }^{1, a)}$ \\ ${ }^{1}$ Department of Physics and Photon Science, School of Physics and Chemistry, Gwangju Institute of Science \\ and Technology, Gwangju 500-712, Korea \\ ${ }^{2}$ Department of Physics and Graphene Research Institute, Sejong University, Seoul 143-747, Korea \\ ${ }^{3}$ Department of Physics, Korea University, Seoul 136-701, Korea \\ ${ }^{4}$ Department of Physics, University or Seoul, Seoul 130-743, Korea \\ ${ }^{5}$ Department of Applied Physics, College of Applied Science, Kyung Hee University, Yongin 446-701, Korea \\ ${ }^{6}$ Department of Electrical and Computer Engineering, Rutgers, The state University of New Jersey, \\ Piscataway, New Jersey 08854, USA \\ ${ }^{7}$ Department of Physics and Astronomy, Rutgers, The state University of New Jersey, Piscataway, \\ New Jersey 08854, USA \\ ${ }^{8}$ Department of Physics, Pohang University of Science and Technology, Pohang 790-784, Korea
}

(Received 16 November 2015; accepted 25 January 2016; published online 4 February 2016)

\begin{abstract}
We investigate the surface and interface states of $\mathrm{Bi}_{2} \mathrm{Se}_{3}$ thin films by using the second-harmonic generation technique. Distinct from the surface of bulk crystals, the film surface and interface show the isotropic azimuth dependence of second-harmonic intensity, which is attributed to the formation of randomly oriented domains on the in-plane. Based on the nonlinear susceptibility deduced from the model fitting, we determine that the surface band bending induced in a space charge region occurs more strongly at the film interface facing the $\mathrm{Al}_{2} \mathrm{O}_{3}$ substrate or capping layer compared with the interface facing the air. We demonstrate that distinct behavior of the terahertz electric field emitted from the samples can provide further information about the surface electronic state of $\mathrm{Bi}_{2} \mathrm{Se}_{3}$. C 2016 AIP Publishing LLC. [http://dx.doi.org/10.1063/1.4941420]
\end{abstract}

The surface state of topological insulators (TIs) has attracted significant attention because it provides a topologically protected and spin-polarized Dirac band; thus, it can serve as a platform for spintronic applications. ${ }^{1-6}$ In particular, thin-film TIs have been highlighted to reduce the bulk carrier effect and also to exploit the surface charge transport due to their large surface-to-volume ratio. The Dirac dispersion of the surface state and the corresponding carrier dynamics were examined by using angle-resolved photoemission and terahertz (THz) spectroscopy, respectively. ${ }^{2-4,7-10}$ In such TIs prepared in the thin-film form, top and bottom interfaces can have distinct electronic behavior, as they face the air and the substrate, for example. Although the Dirac nature of the interface electronic state was examined through tunneling spectroscopy in a TI/non-TI $\mathrm{p}-\mathrm{n}$ junction, it is generally difficult to examine the electronic properties of the bottom interface compared with the top surface. ${ }^{11}$

As a lowest-order-nonlinear-optical process, secondharmonic generation (SHG) can provide valuable information about the surface state of TIs. Second-harmonic ( $\mathrm{SH}$ ) light is generated only in a region with broken inversion symmetry. Since TIs, such as $\mathrm{Bi}_{2} \mathrm{Se}_{3}$, are centrosymmetric, the SHG process takes place only at its surface with longitudinal field discontinuity and in the space charge region with a built-in electric field. Azimuth-dependent SH intensity for the $\mathrm{Bi}_{2} \mathrm{Se}_{3}$ single crystal revealed the crystal symmetry and the time evolution of the space charge region after the cleavage of the sample. ${ }^{12,13}$ Additionally, an attempt was made to

${ }^{a)}$ Electronic mail: jsl@gist.ac.kr understand the spin-momentum-locked state by activating the SHG process with circularly polarized light. ${ }^{12,13}$

In this paper, we investigate the interface electronic states of $\mathrm{Bi}_{2} \mathrm{Se}_{3}$ films as well as their surface states by using the SHG technique. We observe the isotropic azimuth dependence of the SH intensity for $\mathrm{Bi}_{2} \mathrm{Se}_{3}$ films, which we attribute to the signature of the multi-domain formation at both the top surface and the bottom interface of films. Furthermore, we determine part of the second-order susceptibility tensor components from the model fitting of SHG responses and demonstrate that the symmetry breaking is stronger near the interface facing the $\mathrm{Al}_{2} \mathrm{O}_{3}$ layer than the interface facing the air. We also demonstrate that $\mathrm{THz}$ emission spectroscopy can provide complimentary information about the surface electronic state of $\mathrm{Bi}_{2} \mathrm{Se}_{3}$ thin films.

$\mathrm{Bi}_{2} \mathrm{Se}_{3}$ films were fabricated by using commercial molecular beam epitaxy equipment. The substrates used for the film growth are non-crystalline $\mathrm{Si}$ nanocrystals $(\mathrm{Si} \mathrm{NCs}) / \mathrm{Si}$ and crystalline $\mathrm{Al}_{2} \mathrm{O}_{3} \cdot{ }^{14-18}$ For the $\mathrm{Si} \mathrm{NCs} / \mathrm{Si}$ substrate, $\mathrm{SiO}_{\mathrm{x}} / \mathrm{SiO}_{2}$ multilayers were grown on $\mathrm{Si}$ wafers through ion beam sputtering and subsequently annealed to form Si NCs, and then 30-nm-thick $\mathrm{Bi}_{2} \mathrm{Se}_{3}$ was grown. ${ }^{14-16,18}$ For the film grown on $\mathrm{Al}_{2} \mathrm{O}_{3}$, one sample was left with $\mathrm{Bi}_{2} \mathrm{Se}_{3}$ as a top surface, and the other sample was capped with a 20-nm-thick $\mathrm{Al}_{2} \mathrm{O}_{3}$ layer via atomic layer deposition. In the former and latter cases, the $\mathrm{Bi}_{2} \mathrm{Se}_{3}$ film thicknesses were about 30 and $20 \mathrm{~nm}$, respectively. Figure 1(a) shows the x-ray diffraction patterns of $\mathrm{Bi}_{2} \mathrm{Se}_{3}$ films grown on $\mathrm{Si} \mathrm{NCs} / \mathrm{Si}$ (black) and $\mathrm{Al}_{2} \mathrm{O}_{3}$ without (red) and with (blue) the capping layer. They exhibit diffraction peaks corresponding to (003)-type lattice 


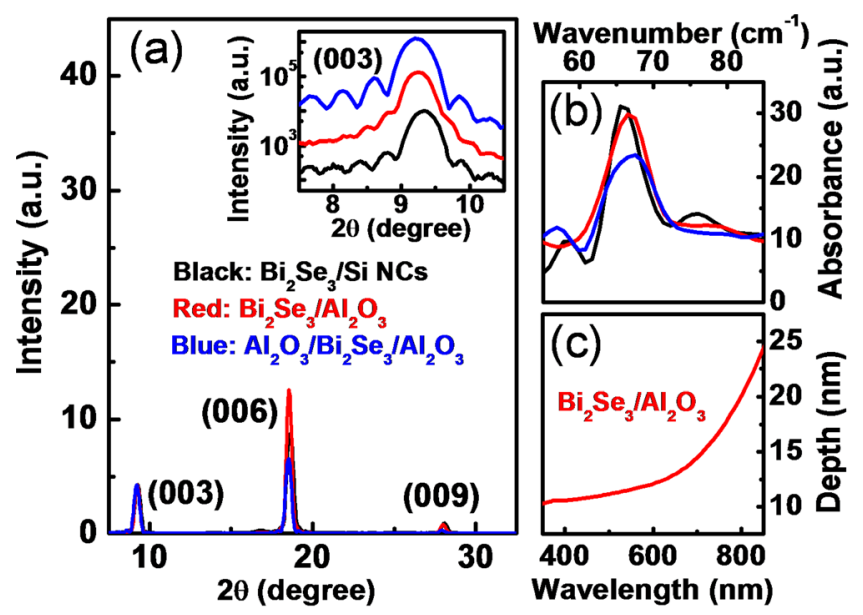

(d)

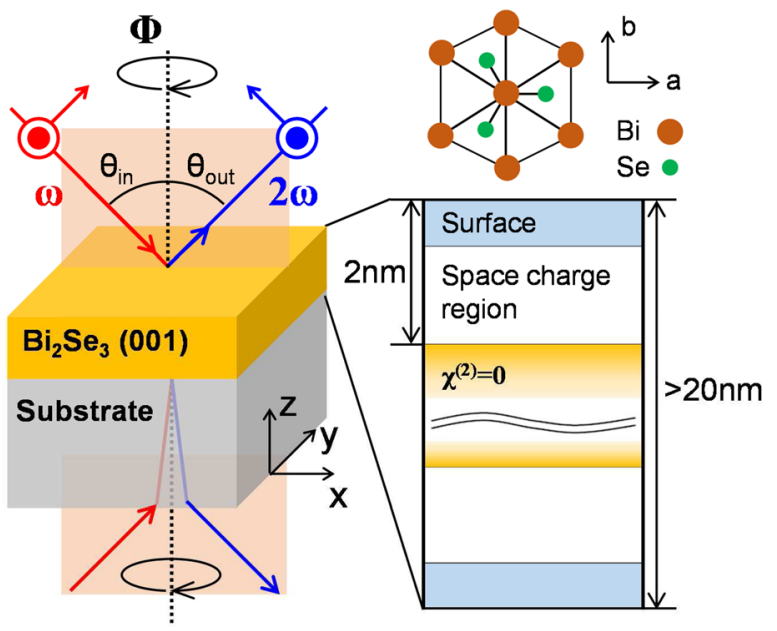

FIG. 1. (a) X-ray diffraction peaks of $\mathrm{Bi}_{2} \mathrm{Se}_{3}$ films. Inset displays an enlarged (003) peak that clearly shows an interference fringe. (b) Absorbance spectra where a transverse optic phonon mode appears as a peak structure at around $65 \mathrm{~cm}^{-1}$. (c) Penetration depth of incident light to $\mathrm{Bi}_{2} \mathrm{Se}_{3}$ thin film. (d) Scheme of second-harmonic generation (SHG) experiment and SHG active regions in the $\mathrm{Bi}_{2} \mathrm{Se}_{3}$ film.

planes and well-defined fringe oscillation around the Bragg peak (inset), which are indicative of an epitaxial growth of the film along the c-axis. Figure 1(b) displays absorbance spectra where a transverse-optic phonon mode appears as a peak structure at around $65 \mathrm{~cm}^{-1}$, which is in close agreement with the reported value of the bulk $\mathrm{Bi}_{2} \mathrm{Se}_{3} .{ }^{19}$ Films with smaller thicknesses down to $6 \mathrm{~nm}$ were prepared, and structural characterization revealed that they have comparable crystallinity compared with thick films. For the comparison, we prepared a single crystal of $\mathrm{Bi}_{2} \mathrm{Se}_{3}$ by using a flux method. All the samples were kept in the air for more than one week before the measurement so that the surface state of $\mathrm{Bi}_{2} \mathrm{Se}_{3}$ could interact sufficiently with an atmospheric environment. $^{20-22}$

We used an 800-nm wavelength wave $(\omega)$ as fundamental light and detected 400-nm wavelength SH light $(2 \omega)$ in a specular-reflection geometry in which the incident and detection angles $\left(\theta_{\text {in }}\right.$ and $\theta_{\text {out }}$, respectively) were $45^{\circ}$ from the surface normal, as shown in Fig. 1(d). Laser pulses of about 100 -fs duration and an $80-\mathrm{MHz}$ repetition rate illuminated the sample with a power density of about $0.1 \mathrm{~kW} / \mathrm{cm}^{2}$, which is lower than the damage threshold. ${ }^{12,13}$ We employed a short-pass filter and a band-pass filter to isolate the SH light emitted from the sample and detected the SH light intensity by using a photomultiplier tube with full variations of input and output polarization as well as the sample azimuth $\Phi$.

The SHG response can be a direct probe of the symmetry breaking selectively at the surface or at the interface depending on the optical geometry. $\mathrm{In}_{\mathrm{Bi}_{2}} \mathrm{Se}_{3}$ films, the interface and the space charge layer, as shown in Fig. 1(d), are the SHG active parts, whereas the inner region is SHGinactive, as it is centrosymmetric. This SHG-active region is about 2-nm thick. ${ }^{12}$ Considering that the penetration depths of light with the wavelengths of 800 and $400 \mathrm{~nm}$ are around 20 and $10 \mathrm{~nm}$, respectively (Fig. $1(\mathrm{c})),{ }^{13}$ the 20 and $30-\mathrm{nm}$ thick films allow a separate probe of surface and interface states by illuminating light from the top and the bottom sides, respectively, and detecting SH light in a backward geometry, as depicted in Fig. 1(d). Note that $\mathrm{Al}_{2} \mathrm{O}_{3}$ is transparent in near-infrared and visible spectral regions, including the wavelengths of 800 and $400 \mathrm{~nm}$, and gives negligible SH intensity.

Figures 2(a)-2(d) display $\Phi$-dependent SH light intensities $I(2 \omega)$ for one single crystal and the other three thin films of $\mathrm{Bi}_{2} \mathrm{Se}_{3}$. All the results were obtained in a $\mathrm{S}_{\text {in }} \mathrm{P}_{\text {out }}$ geometry (i.e., with $\mathrm{S}$-polarized fundamental light and P-polarized $\mathrm{SH}$ light). The bulk $\mathrm{Bi}_{2} \mathrm{Se}_{3}$ shows a three-fold symmetry, which is a typical $3 \mathrm{~m}$ point group response
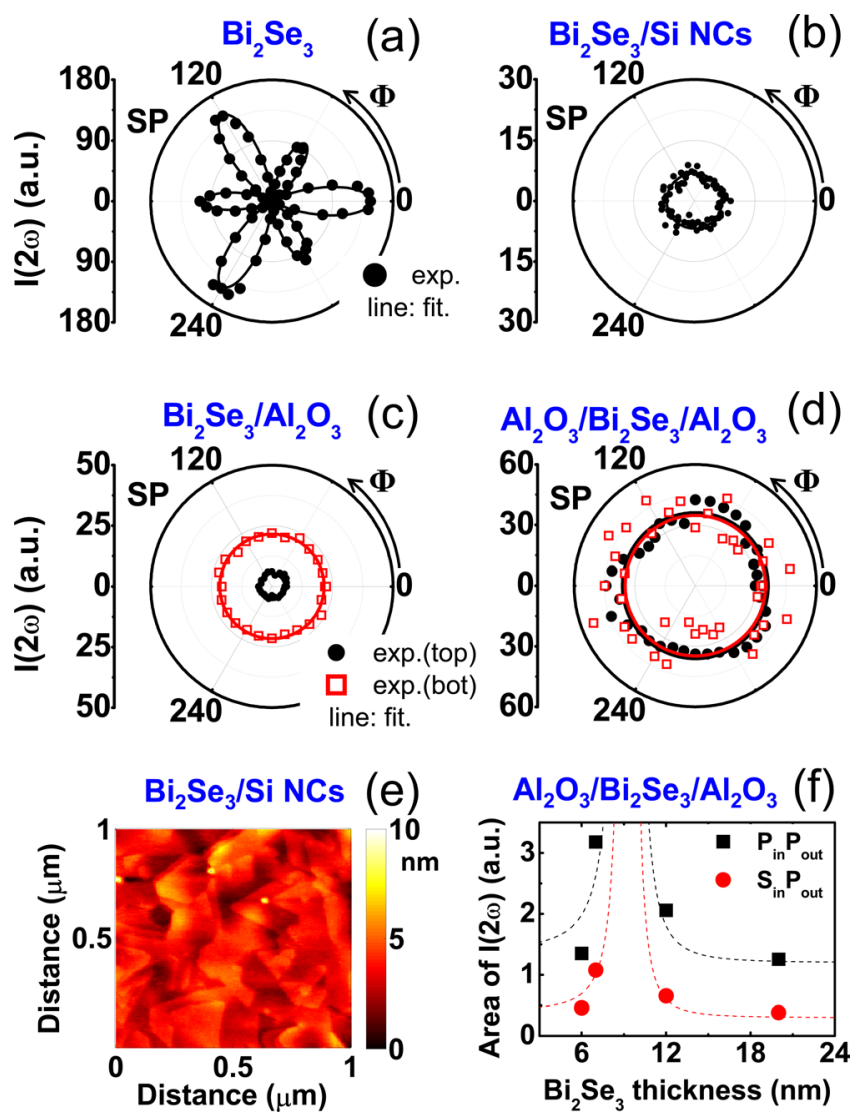

FIG. 2. (a)-(d) Second-harmonic (SH) light intensity $I(2 \omega)$ as function of azimuth angle $\Phi$. SP indicates S- and P-polarization of fundamental and $\mathrm{SH}$ light, respectively. (e) Atomic force microscopy image of $\mathrm{Bi}_{2} \mathrm{Se}_{3}$ films grown on Si NCs/Si. (f) Thickness dependence of $I(2 \omega)$ integrated over $\Phi$. Dotted lines are drawn by considering contributions from capacitor-type dc electric-field and Lorentz-type resonance near $9 \mathrm{~nm}$ to $I(2 \omega)$ in thin films (Ref. 26). 
(Fig. 2(a)). ${ }^{12}$ On the other hand, $I(2 \omega)$ from all three films are finite but unchanged upon the variation of $\Phi$, as shown in Figs. 2(b)-2(d). A similar isotropic SH response is observed for a $\mathrm{P}_{\text {in }} \mathrm{P}_{\text {out }}$ geometry, whereas $I(2 \omega)$ is almost negligible for the $\mathrm{S}_{\text {in }} \mathrm{S}_{\text {out }}$ and $\mathrm{P}_{\text {in }} \mathrm{S}_{\text {out }}$ geometries (not shown).

Although such behavior of $I(2 \omega)$ for the films is reminiscent of the $4 \mathrm{~mm}$ point group, we attribute it instead to the formation of the domains with random in-plane orientations, ${ }^{17,18,23-25}$ this can make the in-plane SH response effectively inversion-symmetric, whereas the inversion symmetry along the out-of-plane direction remains broken. In this context, it is straightforward to understand not only the isotropic SHG responses for $\mathrm{P}_{\text {out }}$ geometries but also the negligible $I(2 \omega)$ for $\mathrm{S}_{\text {out }}$ geometries. The formation of multi-domains is confirmed from the atomic force microscopy images taken for $\mathrm{Bi}_{2} \mathrm{Se}_{3} / \mathrm{Si} \mathrm{NCs}$ (Fig. 2(e)) and $\mathrm{Bi}_{2} \mathrm{Se}_{3} / \mathrm{Al}_{2} \mathrm{O}_{3}$ (not shown). It should be noted that the bottom interface of $\mathrm{Bi}_{2} \mathrm{Se}_{3}$ grown on $\mathrm{Al}_{2} \mathrm{O}_{3}$ exhibited essentially the same isotropic behavior of SH responses (open squares in Fig. 2(c)). Therefore, we suppose that similar domains would also be formed at the film-substrate interface.

Recently, Glinka et al. reported the SHG responses of thin-film TIs with various thicknesses, which showed a clear three-fold symmetry at least for the samples with a thickness of up to $20 \mathrm{~nm} .{ }^{26}$ Such distinct SHG responses between the previous report and this work are attributable to the distribution of domains; supposing that a nonlinear medium is inhomogeneous, $I(2 \omega)$ should depend strongly on the length scale of the inhomogeneity and the spatial coherence of fundament light. Nevertheless, it is worth noting that the thicknessdependent $I(2 \omega)$ exhibits similar behavior to the results in Ref. 26 (Fig. 2(f)).

For the more quantitative analysis, we modeled the induced SH polarization $P(2 \omega)$ as a linear summation of the contributions from each domain as $P(2 \omega)=f \times P_{1}(2 \omega)$ $+(1-f) \times P_{2}(2 \omega)$. Here, $f$ and $(1-f)$ mean the volume fractions of domains 1 and 2 , respectively. In the $\mathrm{S}_{\text {in }} \mathrm{P}_{\text {out }}$ configuration, the $\Phi$-dependent $P(2 \omega)$ is described in the case of the $3 \mathrm{~m}$ point group as $P_{i}^{S_{\text {in }} P_{\text {out }}}(2 \omega)=c_{i}^{(1)} \cos (3 \Phi) a_{i}^{(1)}$ $+c_{i}^{(2)} a_{i}^{(2)}$ with $i=1$ or $2 .{ }^{13} c_{i}^{(1)}$ and $c_{i}^{(2)}$ are constants determined by Fresnel equations with consideration of multiple reflections as, for example, $0.0001+0.0178 i$ and $0.0455-0.0279 i$, respectively, for the surface facing the air. $a_{i}^{(1)}$ and $a_{i}^{(2)}$ are defined as an addition of second- and third-order susceptibilities with the same symmetry (i.e., $a_{i}^{(1)}$ $=\chi_{x x x}+\chi_{x x x z}$ and $\left.a_{i}^{(2)}=\chi_{z x x}+\chi_{z x x z}\right){ }^{13}$ For the multidomains, we assume that there always exist domains with opposite in-plane orientations, and consequently, all the inplane components will be cancelled out. Hence, the polarization formula is changed to $P^{S_{\text {in }} P_{\text {out }}}(2 \omega)=c^{(2)} a^{(2)}$. Here, the subscript for denoting the domain is not used anymore. We fit the experimental data with $I(2 \omega) \propto|P(2 \omega)|^{2}$, as shown by the solid lines in Fig. 2. (The results in Fig. 2(d) have finite three-fold modulation, but we traced the median level to estimate the $a^{(2)}$ value.) For the bulk sample, we first determined $a^{(1)}$ by fitting $I(2 \omega)$ in the $\mathrm{S}_{\text {in }} \mathrm{S}_{\text {out }}$ configuration as $P^{S_{\text {in }} S_{\text {out }}}(2 \omega)=c^{(3)} \sin (3 \Phi) a^{(1)}$ with $c^{(3)}$ determined by the
Fresnel equation and then obtained $a^{(2)}$ from $I(2 \omega)$ in the $\mathrm{S}_{\text {in }} \mathrm{P}_{\text {out }}$ configuration using the aforementioned equation. ${ }^{13}$

By investigating the details of fitting parameters, we discuss the degree of inversion symmetry breaking for the film surfaces and interfaces. Figure 3 shows $\left|a^{(2)}\right|$ values obtained for the $\mathrm{Bi}_{2} \mathrm{Se}_{3}$ bulk and thin films. All the quantities are normalized to the value of the bulk sample. Filled circles and open squares denote parameters for the top and the bottom $\mathrm{Bi}_{2} \mathrm{Se}_{3}$ interfaces, respectively. Interestingly, all the surfaces facing the air have comparable values of $\left|a^{(2)}\right|$ irrespective of the sample types or substrates used for the film growth. It is worth emphasizing that the value of the interface facing the $\mathrm{Al}_{2} \mathrm{O}_{3}$ layer is larger by about four times than those of the interfaces between $\mathrm{Bi}_{2} \mathrm{Se}_{3}$ and air. This demonstrates that the inversion symmetry is broken more strongly in the former case than the latter.

Since the SHG can occur at the surface itself or in the space charge region, it is unclear which one has the dominant contribution to the larger symmetry breaking at the $\mathrm{Bi}_{2} \mathrm{Se}_{3}-$ $\mathrm{Al}_{2} \mathrm{O}_{3}$ interface; accordingly, a complexity arises in the interpretation of the SHG results. Concerning the relative phase of each contribution to the total SH intensity, on the other hand, we can obtain a useful hint from a report by Hsieh et al. for the $\mathrm{Bi}_{2} \mathrm{Se}_{3}$ single crystal; the time-dependent $I(2 \omega)$ shows a monotonic increase after the sample cleavage. ${ }^{12,13}$ Since the downward band bending progresses due to the Se vacancy, this suggests that the surface charge discontinuity and the downward band bending have the same phase in their contributions to SHG. Note that the charge discontinuity is expected to be less for the $\mathrm{Al}_{2} \mathrm{O}_{3}-\mathrm{Bi}_{2} \mathrm{Se}_{3}$ interface than for the air $-\mathrm{Bi}_{2} \mathrm{Se}_{3}$ interface. Therefore, the larger

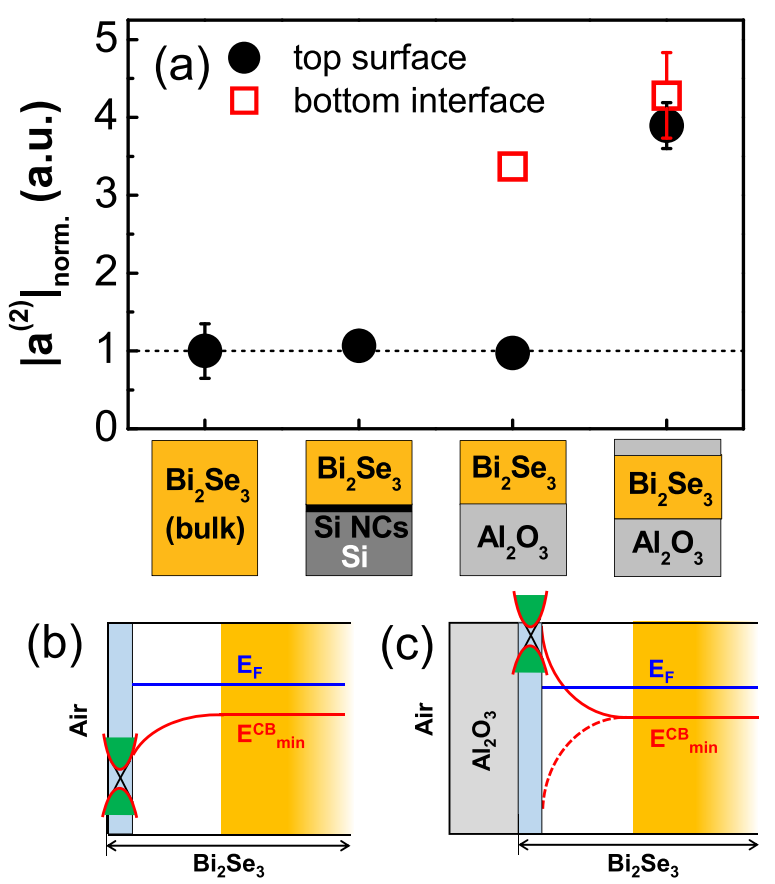

FIG. 3. (a) Sample-dependent second-order nonlinear tensor component $\left|a^{(2)}\right|$ normalized to value for bulk $\mathrm{Bi}_{2} \mathrm{Se}_{3}$. (b) and (c) Schematics of surface band bending of $\mathrm{Bi}_{2} \mathrm{Se}_{3}$ facing the air and $\mathrm{Al}_{2} \mathrm{O}_{3}$, respectively. Region in light blue (orange) indicates surface (bulk) state. $\mathrm{E}_{\mathrm{F}}$ and $\mathrm{E}_{\text {min }}^{\mathrm{CB}}$ represent Fermi energy and conduction band minimum, respectively. Dotted and solid lines in (c) display two possible band bending states at $\mathrm{Bi}_{2} \mathrm{Se}_{3}$ interface facing $\mathrm{Al}_{2} \mathrm{O}_{3}$. 
$\left|a^{(2)}\right|$ for the $\mathrm{Al}_{2} \mathrm{O}_{3}-\mathrm{Bi}_{2} \mathrm{Se}_{3}$ interface compared with that of the air- $-\mathrm{Bi}_{2} \mathrm{Se}_{3}$ interface is attributable to the larger contribution of surface band bending. This interpretation is further reinforced if we assume the destructive contribution of the charge discontinuity against surface band bending. Figures 3(b) and 3(c) depict possible surface electronic states of $\mathrm{Bi}_{2} \mathrm{Se}_{3}$ facing the air and $\mathrm{Al}_{2} \mathrm{O}_{3}$, respectively. Here, the air- $\mathrm{Bi}_{2} \mathrm{Se}_{3}$ interface is considered to have downward bend bending, ${ }^{4,12,13,27}$ and the $\mathrm{Al}_{2} \mathrm{O}_{3}-\mathrm{Bi}_{2} \mathrm{Se}_{3}$ interface provides stronger band bending although its direction has not been determined yet.

As a second probe of the surface electronic states, $\mathrm{THz}$ emission arising from the photo-carrier acceleration or optical rectification is examined. Similarly with the SHG experiment, a THz wave can be generated near the surface upon its illumination with infrared laser pulses (Fig. 4(a)). The emitted $\mathrm{THz}$ wave is guided by a set of parabolic mirrors, and its transient electric field profiles are detected by a photoconductive antenna. ${ }^{28,29}$ The time-profiles of the $\mathrm{THz}$ electric field emitted from the top surfaces of $\mathrm{Bi}_{2} \mathrm{Se}_{3}$ are displayed in Fig. 4(b). Except for the $\mathrm{Bi}_{2} \mathrm{Se}_{3}$ film grown on $\mathrm{Al}_{2} \mathrm{O}_{3}$ without the capping layer, the $\mathrm{THz}$ electric field exhibits isotropic behavior upon the variation of $\Phi$ (not shown). ${ }^{9,29}$ This leads us to exclude optical rectification as a possible mechanism of the $\mathrm{THz}$ emission and to consider the surge current mechanism as a primary emission source of the $\mathrm{THz}$ emission from $\mathrm{Bi}_{2} \mathrm{Se}_{3}$. Upon the formation of photoexcited carriers, a transient electric dipole-generating $\mathrm{THz}$ wave is formed by a mobility difference between an electron and a hole (photo-Dember effect) or by a chargeacceleration due to a built-in field. We take the $\mathrm{THz}$ wave emitted from InAs as a reference waveform induced by the larger electron mobility than the hole (Fig. 4(b)).$^{30}$ When the photo-carriers are accelerated in upward (downward) band bending, the direction of the transient dipole, or equivalently the phase of the THz wave, will be the same as (opposite to) that formed in InAs. ${ }^{31}$ Therefore, we determined the surface electronic states by examining the details of the $\mathrm{THz}$ field profiles, namely, the phase and amplitude of the emitted $\mathrm{THz}$ wave.

For $\mathrm{Bi}_{2} \mathrm{Se}_{3}$ facing the air, the phase of the $\mathrm{THz}$ wave is opposite to that of InAs (Fig. 4(b)). In a recent report by Luo et al., however, the $\mathrm{THz}$ wave emitted from $\mathrm{Bi}_{2} \mathrm{Se}_{3}$ has the same phase as that of InAs. ${ }^{9}$ These contrasting results imply
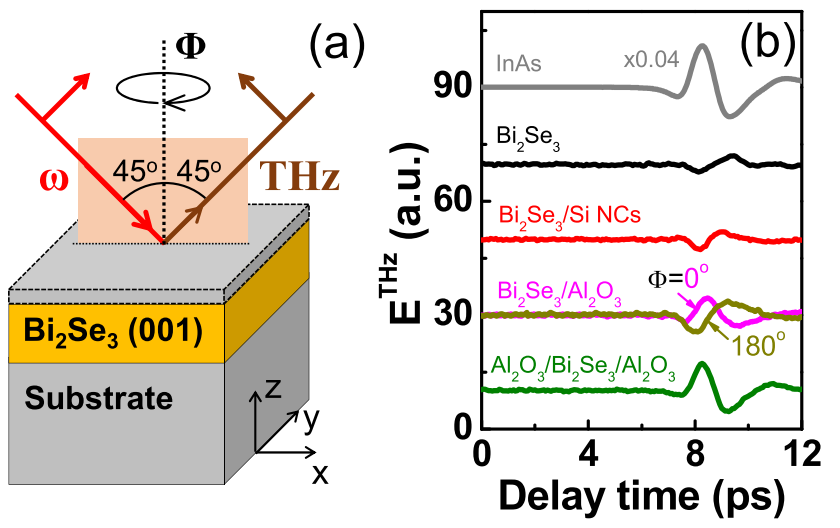

FIG. 4. (a) Schematic of terahertz (THz) emission experiment. (b) Timeprofiles of $\mathrm{THz}$ electric field emitted from top surfaces of $\mathrm{Bi}_{2} \mathrm{Se}_{3}$ samples. that the $\mathrm{THz}$ phase is determined sensitively by the detailed balance between two competing contributions from the mobility difference and the band bending. In the case of the $\mathrm{Bi}_{2} \mathrm{Se}_{3}$ crystal studied in the present work, the opposite phase of the emitted $\mathrm{THz}$ wave compared with that of InAs is attributed to the fact that the dominant $\mathrm{THz}$ generation mechanism at the air- $\mathrm{Bi}_{2} \mathrm{Se}_{3}$ interface is downward band bending (Fig. 3(b)), which is in close agreement with the conventional understanding of the surface state of $\mathrm{Bi}_{2} \mathrm{Se}_{3} \cdot{ }^{4,12,13,27}$ In this way, when the $\mathrm{THz}$ wave has an opposite phase to that of InAs, the direction of the band bending can be clearly determined. However, the $\mathrm{Bi}_{2} \mathrm{Se}_{3}$ film surface facing $\mathrm{Al}_{2} \mathrm{O}_{3}$ is not straightforward, as the phase of the $\mathrm{THz}$ wave is the same as that of InAs. This implies two possible interpretations. Firstly, the surface facing $\mathrm{Al}_{2} \mathrm{O}_{3}$ would emit the $\mathrm{THz}$ wave mainly through the photo-Dember effect. Secondly, the surface state of $\mathrm{Bi}_{2} \mathrm{Se}_{3}$ may have upward band bending so that the photo-Dember effect and the band bending contribute constructively to the large $\mathrm{THz}$ field strength. To reach a clearer conclusion about the surface electronic state, additional efforts are desirable to separate the photo-Dember effect from $\mathrm{THz}$ emission processes.

In summary, we investigated the surface and interface states of $\mathrm{Bi}_{2} \mathrm{Se}_{3}$ thin films in the aspect of inversion symmetry breaking by employing $\mathrm{SHG}$ and $\mathrm{THz}$ emission techniques. We modeled the SHG process by considering the domains with random in-plane orientations to account for the isotropic azimuth dependence of the second-harmonic intensity and estimated part of the second-order-nonlinear susceptibility tensor components. Additionally, we examined electric-field profiles of emitted $\mathrm{THz}$ waves, which showed distinct behavior in their phases depending on the adjacent layer that the $\mathrm{Bi}_{2} \mathrm{Se}_{3}$ film faced. Based on these results, we suggested that inversion symmetry breaking occurs more strongly at the interface facing $\mathrm{Al}_{2} \mathrm{O}_{3}$ than that facing the air. The band bending formed in the space charge region can shift the location of the Fermi energy relative to the surface Dirac state, as depicted schematically in Figs. 3(b) and 3(c), which can determine the majority-carrier type of the surface state as well as the carrier number. Therefore, the transport properties of the interface/surface state of topological insulators can be controlled by choosing a proper substrate or capping layer, and interface-sensitive SHG and $\mathrm{THz}$ emission techniques can provide useful information about interface/ surface electronic states that is otherwise difficult to obtain.

We would like to thank Jae Myung Kim, Okkyun Seo, Do Young Noh, Sungsu Lee, and Ji Young Jo for their contribution to x-ray diffraction measurement. This work was supported in part by the Science Research Center and the Basic Science Research Program through the National Research Foundation of Korea (NRF) funded by the Ministry of Science, ICT and Future Planning (Nos. 2015R1A5A1009962 and 2015R1A1A1A05001560) and also by the Top Brand Project through a grant provided by the Gwangju Institute of Science and Technology in 2015. The work at Sejong was also supported by NRF (Nos. 20100020207, 2011-0030786, and 2014R1A2A2A01005963). The work at Rutgers was supported by Office of Naval Research (N000141210456). The work at POSTECH was 
supported by the NRF through SRC (Grant No. 20110030785) and Max Planck POSTECH/KOREA Research Initiative Programs (Grant No. 2011-0031558).

${ }^{1}$ L. Fu, C. L. Kane, and E. J. Mele, Phys. Rev. Lett. 98, 106803 (2007).

${ }^{2}$ D. Hsieh, D. Qian, L. Wray, Y. Xia, Y. S. Hor, R. J. Cava, and M. Z. Hasan, Nature 452, 970-974 (2008).

${ }^{3}$ D. Hsieh, Y. Xia, L. Wray, D. Qian, A. Pal, J. H. Dil, J. Osterwalder, F. Meier, G. Bihlmayer, C. L. Kane, Y. S. Hor, R. J. Cava, and M. Z. Hasan, Science 323, 919-922 (2009).

${ }^{4}$ D. Hsieh, Y. Xia, D. Qian, L. Wray, J. H. Dil, F. Meier, J. Osterwalder, L. Patthey, J. G. Checkelsky, N. P. Ong, A. V. Fedorov, H. Lin, A. Bansil, D. Grauer, Y. S. Hor, R. J. Cava, and M. Z. Hasan, Nature 460, 1101-1105 (2009).

${ }^{5}$ J. E. Moore, Nature 464, 194-198 (2010).

${ }^{6}$ M. Z. Hasan and C. L. Kane, Rev. Mod. Phys. 82, 3045 (2010).

${ }^{7}$ Y. Zhang, K. He, C. Z. Chang, C. L. Song, L. L. Wang, X. Chen, J. F. Jia, Z. Fang, X. Dai, W. Y. Shan, S. Q. Shen, Q. Niu, X. L. Qi, S. C. Zhang, X. C. Ma, and Q. K. Xue, Nat. Phys. 6, 584-588 (2010).

${ }^{8}$ R. V. Aguilar, A. V. Stier, W. Liu, L. S. Bilbro, D. K. George, N. Bansal, L. Wu, J. Cerne, A. G. Markelz, S. Oh, and N. P. Armitage, Phys. Rev. Lett. 108, 087403 (2012).

${ }^{9}$ C. W. Luo, H. J. Chen, C. M. Tu, C. C. Lee, S. A. Ku, W. Y. Tzeng, T. T. Yeh, M. C. Chiang, H. J. Wang, W. C. Chu, J. Y. Lin, K. H. Wu, J. Y. Juang, T. Kobayashi, C. M. Cheng, C. H. Chen, K. D. Tsuei, H. Berger, R. Sankar, F. C. Chou, and H. D. Yang, Adv. Opt. Mater. 1(11), 804-808 (2013).

${ }^{10}$ B. C. Park, T.-H. Kim, K. I. Sim, B. Kang, J. W. Kim, B. Cho, K.-H. Jeong, M.-H. Cho, and J. H. Kim, Nat. Commun. 6, 6552 (2015).

${ }^{11}$ R. Yoshimi, A. Tsukazaki, K. Kikutake, J. G. Checkelsky, K. S. Takahashi, M. Kawasaki, and Y. Tokura, Nat. Mater. 13, 253-257 (2014).

${ }^{12}$ D. Hsieh, J. W. McIver, D. H. Torchinsky, D. R. Gardner, Y. S. Lee, and N. Gedik, Phys. Rev. Lett. 106, 057401 (2011).

${ }^{13}$ J. W. McIver, D. Hsieh, S. G. Drapcho, D. H. Torchinsky, D. R. Gardner, Y. S. Lee, and N. Gedik, Phys. Rev. B 86, 035327 (2012).

${ }^{14}$ N. Bansal, Y. S. Kim, E. Edrey, M. Brahlek, Y. Horibe, K. Iida, M. Tanimura, G. H. Li, T. Feng, H. D. Lee, T. Gustafsson, E. Andrei, and S. Oh, Thin Solid Films 520, 224-229 (2011).

${ }^{15}$ K. J. Kim, D. W. Moon, S. H. Hong, S. H. Choi, M. S. Yang, J. H. Jhe, and J. H. Shin, Thin Solid Films 478, 21-24 (2005).

${ }^{16}$ S. Kim, M. C. Kim, S. H. Choi, K. J. Kim, H. N. Hwang, and C. C. Hwang, Appl. Phys. Lett. 91, 103113 (2007).
${ }^{17}$ S.-K. Jerng, K. Joo, Y. Kim, S.-M. Yoon, J. H. Lee, M. Kim, J. S. Kim, E. Yoon, S.-H. Chun, and Y. S. Kim, Nanoscale 5, 10618 (2013).

${ }^{18}$ N. Bansal, N. Koirala, M. Brahlek, M.-G. Han, Y. Zhu, Y. Cao, J. Waugh, D. S. Dessau, and S. Oh, Appl. Phys. Lett. 104, 241606 (2014).

${ }^{19}$ A. D. LaForge, A. Frenzel, B. C. Pursley, T. Lin, X. Liu, J. Shi, and D. N. Basov, Phys. Rev. B 81, 125120 (2010).

${ }^{20}$ M. Brahlek, Y. S. Kim, N. Bansal, E. Edrey, and S. Oh, Appl. Phys. Lett. 99, 012109 (2011).

${ }^{21}$ C. Chen, S. He, H. Weng, W. Zhang, L. Zhao, H. Liu, X. Jia, D. Mou, S. Liu, J. He, Y. Peng, Y. Feng, Z. Xie, G. Liu, X. Dong, J. Zhang, X. Wang, Q. Peng, Z. Wang, S. Zhang, F. Yang, C. Chen, Z. Xu, X. Dai, Z. Fang, and X. J. Zhou, Proc. Natl. Acad. Sci. U. S. A. 109(10), 3694-3698 (2012).

${ }^{22}$ L. V. Yashina, J. Sanchez-Barriga, M. R. Scholz, A. A. Volykhov, A. P. Sirotina, V. S. Neudachina, M. E. Tamm, A. Varykhalov, D. Marchenko, G. Springholz, G. Bauer, A. Knop-Gericke, and O. Rader, ACS Nano 7(6), 5181-5191 (2013).

${ }^{23}$ D. L. Medlin, Q. M. Ramasse, C. D. Spataru, and N. Y. C. Yang, J. Appl. Phys. 108, 043517 (2010).

${ }^{24}$ N. V. Tarakina, S. Schreyeck, T. Borzenko, C. Schumacher, G. Karczewski, K. Brunner, C. Gould, H. Buhmann, and L. W. Molenkamp, Cryst. Growth Des. 12(4), 1913-1918 (2012).

${ }^{25}$ S. Schreyeck, N. V. Tarakina, G. Karczewski, C. Schumacher, T. Borzenko, C. Brüne, H. Buhmann, C. Gould, K. Brunner, and L. W. Molenkamp, Appl. Phys. Lett. 102, 041914 (2013).

${ }^{26}$ Y. D. Glinka, S. Babakiray, T. A. Jhhnson, M. B. Holcomb, and D. Lederman, Phys. Rev. B 91, 195307 (2015).

${ }^{27}$ H.-J. Noh, H. Koh, S.-J. Oh, J.-H. Park, H.-D. Kim, J. D. Rameau, T. Valla, T. E. Kidd, P. D. Johnson, Y. Hu, and Q. Li, Europhys. Lett. 81, 57006 (2008).

${ }^{28}$ J. W. Han, S. Y. Hamh, T. H. Kim, K. S. Lee, N. E. Yu, D.-K. Ko, and J. S. Lee, Opt. Lett. 39(19), 5531-5534 (2014).

${ }^{29} \mathrm{The}^{\mathrm{Bi}} \mathrm{Se}_{3}$ film grown on $\mathrm{Al}_{2} \mathrm{O}_{3}$ without the capping layer exhibits an anisotropic behaviour which was attributed to the vicinality of the substrate and inclined film surface. (S. Y. Hamh et al., Nanoscale Res. Lett. 10, 489 (2015).) We observed a similar anisotropic azimuth-dependence for the bottom interface formed between $\mathrm{Bi}_{2} \mathrm{Se}_{3}$ film and the $\mathrm{Al}_{2} \mathrm{O}_{3}$ substrate, and excluded it in the discussion.

${ }^{30}$ Y. B. Gao, B. He, D. Parker, I. Androulakis, and J. P. Heremans, Phys. Rev. B 90, 125204 (2014).

${ }^{31}$ P. Gu, M. Tani, S. Kono, K. Sakai, and X. C. Zhang, J. Appl. Phys. 91, 5533 (2002). 